\title{
Teaching and Learning Electromagnetic Simulation by Means of a Two-Dimensional Finite-Difference Time-Domain Method
}

\author{
Alejandro Dueñas Jiménez \\ Universidad de Guadalajara \\ *Corresponding Author: alejandro.duenas@red.cucei.udg.mx
}

Copyright (C) 2013 Horizon Research Publishing All rights reserved.

\begin{abstract}
A proposal for teaching and learning electromagnetic simulation by means of a two-dimensional finite-difference time-domain method is presented. The suggestion has been well received by the academic community since the teaching material is easy to implement and simple to explain in a classroom environment. Two specific codes to simulate microstrip printed dipoles are presented in here but many more can be implemented by following the book references. In addition, the running times are fast enough as to include several examples in each class session.
\end{abstract}

Keywords Electromagnetic Simulation, FDTD Method, Lengthening Factor, Microstrip Circuits, Propagation Speed, Resonance

\section{Introduction}

No matter the knowledge to be transmitted, the process of teaching and learning should be a simple task. Although the electromagnetic theory is the basis of all the electromagnetic analysis methods, some of them are more difficult to understand, implement and manage as compared to others. Likewise, some methods are more appropriates to solve complex problems, thereby when someone use it to carry out simple tasks it seems like trying to kill flies with cannon. Thus for instance, the method of moments (MoM) is based on a solid mathematical model that warranties results close to the circuit real behaviors; however, for a non graduated student or for a novice practitioner, the waiting for large time periods to obtain a simple response could be frustrating, even if the method had been optimized by means of some kind of frequency adaptive sampling, to reduce the time of matrix filling and inversion. In this way, to efficiently teach and learn the art of the electromagnetic simulation is better to initiate with a non complicated method that can be easily implemented in homemade open codes. The two-dimensional finite difference time domain method (2D-FDTD), amalgam all these qualities. Thus, once the novel apprentices manage adequately every basic concept of the FDTD method, then they can confidently pass to use the commercial professional software.

In this context, the codes presented here are not intended to replace the powerful simulation packages, but to help attain a better comprehension and manage of the commercial ones. Three microstrip printed dipoles are used as examples to implement Matlab $^{\circledR}$ codes where the geometries are defined by means of code lines. Two main programs are prepared by following the general procedure described in [1]. In each program some extra code lines are added to meet the specific requirements of each dipole. In the first program, the normalization proposed in [2] is considered. In the second program, the free space impedance and the far enclosure covers are taken into account. This kind of codes has been extensively used to teach FDTD electromagnetic simulations and the instructors had reported a notable profiting on the student's learning.

As compared to traditional analyses carried out by means of the classical circuit and network theories, the electromagnetic simulation has two notable advantages: to be intrinsic in its nature and robust as a visual tool. Additionally, as a student's generalized opinion, the learning by means of the electromagnetic simulations creates an environment of confidence and security through an entire course.

\section{The Codes}

Two printed wire dipoles are simulated by calling the Code1 and a printed planar dipole is simulated by calling the Code2. The radii of the wire dipoles are very small as compared to the free space wavelength; hence an approximation considering thin or planar wires can be used. The dipoles are impressed on top of a grounded dielectric slice considered as a simple microstrip. Both codes call the function "miconedinolo" to calculate the inductance and 
capacitance per unit length, the characteristic impedance and the phase velocity on a microstrip considering neither the dispersion nor the losses [4], [5]. The phase velocity is corrected with the lengthening factor $\left(f_{l}\right)$ to obtain the correct resonant frequency $[6,7]$. This factor is expressed in terms of the microstrip phase velocity $v_{p m}$ (which is taken as the velocity on the printed dipole) and the phase velocity on the dielectric $v_{p d}$, in the following way [1]:

$$
f_{l}=\frac{k v_{p m}^{n}}{v_{p d}^{n}}
$$

where

$$
\begin{gathered}
v_{p m}=\frac{c}{\sqrt{\varepsilon_{e f f}}}=c \frac{1}{\sqrt{\varepsilon_{e f f}}}=c v_{r p} \\
v_{p d}=\frac{c}{\sqrt{\varepsilon_{r}}}
\end{gathered}
$$

$k$ and $n$ are positive numbers, $k=p / 2$ (where $p$ is the number of ports in the circuit), $n$ is experientially chosen as a positive integer number, $c$ is the free space speed of light, $\varepsilon_{e f f}$ is the effective dielectric constant, $v_{r p}$ is the relative phase velocity and $\varepsilon_{r}$ is the relative permittivity.

Thus,

$$
v_{p m c}=v_{p m} \cdot f_{l}
$$

where $f_{l}$ is given by (1) for $k=n=1$.

To analyze a printed dipole with the MoM procedure, it is necessary to assume a surface resistivity of $377 \mathrm{Ohms}$ per square on the upper and lower covers (ground plane) of the box containing the circuit, and remove them a distance of at least a half wavelength [3]. The correspondent with the 2D-FDTD technique described here is to move the ground plane a similar distance through an increment on the microstrip substrate thickness $\mathrm{H}$, which will become in a microstrip width $\mathrm{W}$ to thickness $\mathrm{H}(\mathrm{W} / \mathrm{H})$ relation, out of the recommended limits for a typical microstrip. The increment in the substrate thickness corresponds to an increment in the microstrip characteristic impedance, which should be a considerable fraction of the free space impedance. By following these directions, the two main programs and the function subroutine can be written as follows:

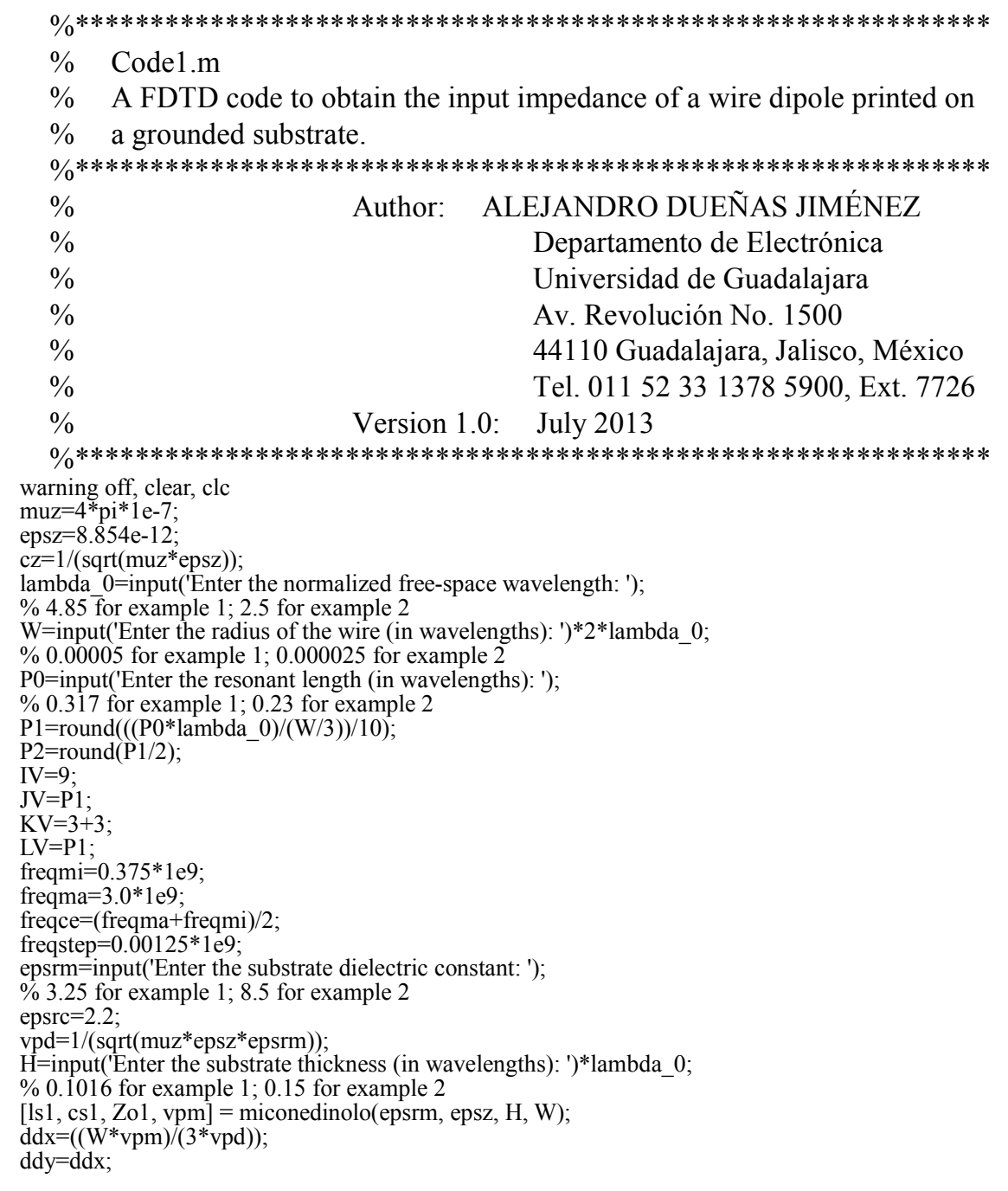




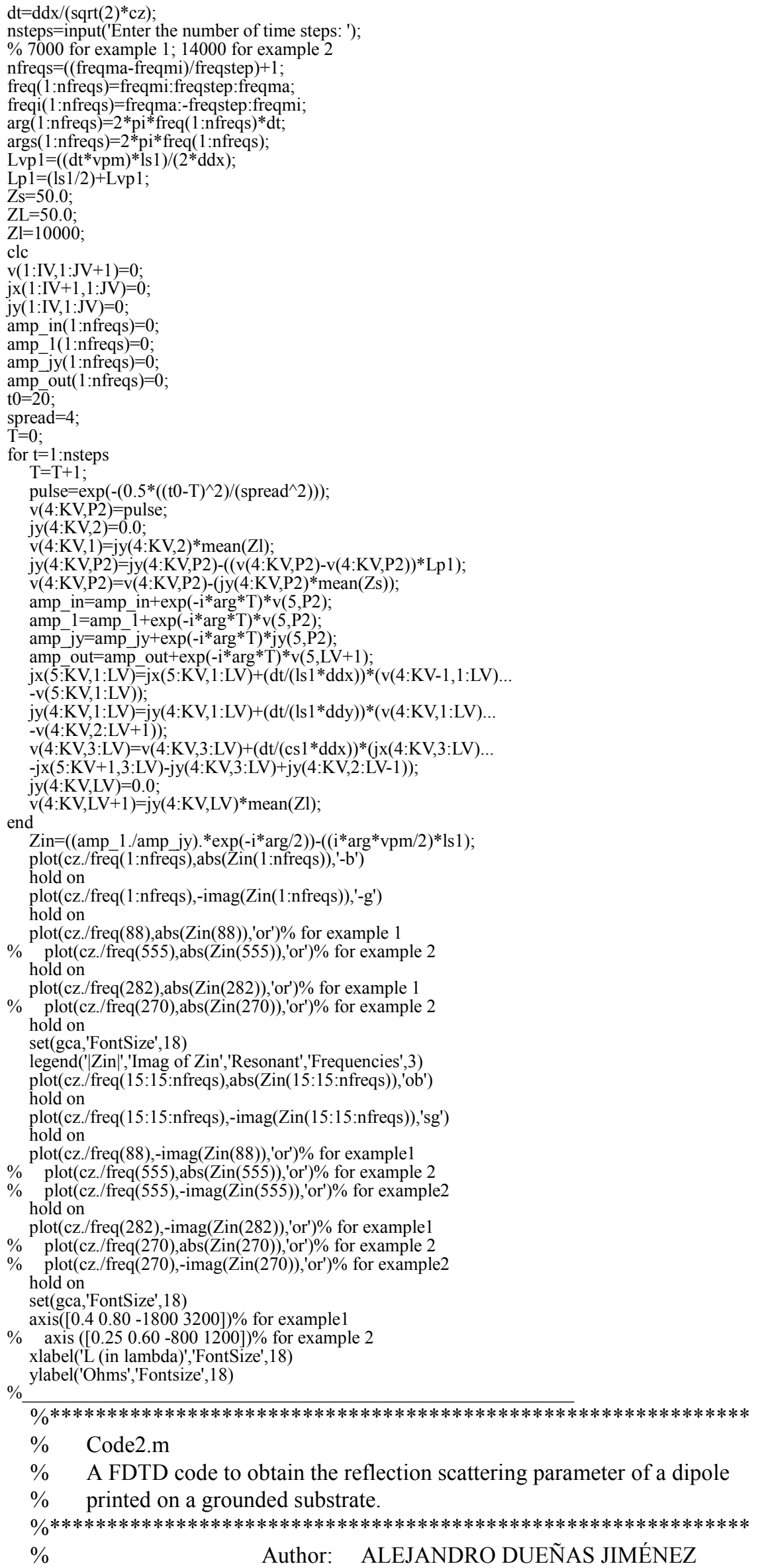




\begin{tabular}{|c|c|c|}
\hline$\%$ & & Departamento de Electrónica \\
\hline$\%$ & & Universidad de Guadalajara \\
\hline$\%$ & & Av. Revolución No. 1500 \\
\hline$\%$ & & 44110 Guadalajara, Jalisco, México \\
\hline$\%$ & & Tel. 01152331378 5900, Ext. 7726 \\
\hline$\%$ & Version 1.0: & July 2013 \\
\hline
\end{tabular}

warning off, clear, clc

ADJ=input('Enter the sweep of the dipole length: ');

$\% 3 ; 6 ; 9 ; 12 ; 15 ; 18 ; 21 ; 24 ; 27 ; 30$

$\mathrm{IV}=9$;

$\mathrm{JV}=186+\mathrm{ADJ}$

$\mathrm{KV}=3+3$;

$\mathrm{LV}=186+\mathrm{ADJ}$

freqmi $=0.0 * 1 \mathrm{e} 9$;

freqma $=1.2 * 1 \mathrm{e} 9$;

freqce $=($ freqma + freqmi $) / 2$;

freqstep $=0.001 * 1 \mathrm{e} 9$;

epsrm $=3.0$;

muz $=4 * p^{*} i^{*} 1 \mathrm{e}-7$;

epsz $=8.854 \mathrm{e}-12$;

epsrc $=2.2$;

$\mathrm{c} z=1 /(\operatorname{sqrt}(\mathrm{muz} * \mathrm{epsz}))$;

freqre $=0.954 * 1 \mathrm{e} 9$;

vpd $=1 /\left(\operatorname{sqrt}\left(\right.\right.$ muz $^{*}$ epsz*epsrm $)$ );

$\mathrm{H}=$ input('Enter the normalized substrate thickness: ');

$\% 0.8$

$\mathrm{W}=0.002$;

$[1 \mathrm{~s} 1, \mathrm{cs} 1, \mathrm{Zo1}, \mathrm{vpm}]=$ miconedinolo $($ epsrm, epsz, $\mathrm{H}, \mathrm{W})$;

$\% \mathrm{~W} / \mathrm{H}$ Recommended $>=0.05$

$\mathrm{ddx}=\left(\left(\mathrm{W}^{*} \mathrm{vpm}\right) /\left(3^{*} \mathrm{vpd}\right)\right)$;

$\mathrm{ddy}=\mathrm{ddx}$;

$\mathrm{dt}=\mathrm{ddx} /(\operatorname{sqrt}(2) * \mathrm{cz})$;

nsteps=input('Enter the number of time steps: ');

$\% 2000$ for example 3

nfreqs $=(($ freqma-freqmi $) /$ freqstep $)+1$;

freq $(1:$ nfreqs $)=$ freqmi:freqstep:freqma;

freqi $(1:$ nfreqs $)=$ freqma:-freqstep:freqmi;

$\arg (1:$ nfreqs $)=2 *$ pi $*$ freq $(1:$ nfreqs $) * d t ;$

$\operatorname{args}(1$ :nfreqs $)=2 *$ pi*freq $(1:$ nfreqs $)$;

Lvp1 $=((\mathrm{dt} * \mathrm{vpm}) * 1 \mathrm{~s} 1) /(2 * \mathrm{ddx})$

Lp1 $=(1 \mathrm{~s} 1 / 2)+$ Lvp1;

$\mathrm{Zs}=50.0$;

$\mathrm{ZL}=50.0$;

$\mathrm{Zl}=10000$

clc

$\mathrm{v}(1: \mathrm{IV}, 1: \mathrm{JV}+1)=0$;

$\mathrm{jx}(1: \mathrm{IV}+1,1: \mathrm{JV})=0$;

jy $(1: \mathrm{IV}, 1: \mathrm{JV})=0$;

amp_in(1:nfreqs $)=0$;

amp $1(1$ :nfreqs $)=0$;

amp_jy $(1:$ nfreqs $)=0$;

amp_out $(1:$ nfreqs $)=0$;

$\mathrm{t} 0=2 \overline{0}$

spread $=4$;

$\mathrm{T}=0$;

for $\mathrm{t}=1$ :nsteps

$\mathrm{T}=\mathrm{T}+1$;

pulse $=\exp \left(-\left(0.5^{*}\left((\mathrm{t} 0-\mathrm{T})^{\wedge} 2\right) /\left(\operatorname{spread}^{\wedge} 2\right)\right)\right) ;$

$\mathrm{v}(4: \mathrm{KV}, 102)=$ pulse;

$\mathrm{jy}(4: \mathrm{KV}, 2)=0.0$

$\mathrm{v}(4: \mathrm{KV}, 1)=\mathrm{jy}(4: \mathrm{KV}, 2) * \operatorname{mean}(\mathrm{Zl})$;

jy $(4: K V, 102)=j y(4: K V, 102)-((v(4: K V, 102)-v(4: K V, 102)) * L p 1)$;

$\mathrm{v}(4: \mathrm{KV}, 102)=\mathrm{v}(4: \mathrm{KV}, 102)-(\mathrm{jy}(4: \mathrm{KV}, 102) * \operatorname{mean}(\mathrm{Zs}))$;

amp_in=amp in $+\exp (-1 * \arg * \mathrm{~T}) * \mathrm{v}(5,102)$;

amp $1=\operatorname{amp} 1+\exp \left(-i^{*} \arg * \mathrm{~T}\right) * \mathrm{v}(5,102)$;

amp_jy=amp_jy+exp(-i*arg*T)*jy $(5,102)$;

amp_out=amp_out $+\exp (-i * \arg * \mathrm{~T}) * \mathrm{v}(5, \mathrm{LV}+1)$;

$\mathrm{jx}(5: \mathrm{KV}, 1: \mathrm{LV})=\mathrm{jx}(5: \mathrm{KV}, 1: \mathrm{LV})+(\mathrm{dt} /(\mathrm{ls} 1 * \mathrm{ddx})) *(\mathrm{v}(4: \mathrm{KV}-1,1: \mathrm{LV}) \ldots$

-v(5:KV,1:LV));

jy $(4: \mathrm{KV}, 1: \mathrm{LV})=\mathrm{jy}(4: \mathrm{KV}, 1: \mathrm{LV})+(\mathrm{dt} /(\mathrm{ls} 1 \text { *ddy }))^{*}(\mathrm{v}(4: \mathrm{KV}, 1: \mathrm{LV}) \ldots$

-v(4:KV,2:LV+1));

$\mathrm{v}(4: \mathrm{KV}, 3: \mathrm{LV})=\mathrm{v}(4: \mathrm{KV}, 3: \mathrm{LV})+(\mathrm{dt} /(\mathrm{cs} 1 * \mathrm{ddx})) *(\mathrm{jx}(4: \mathrm{KV}, 3: \mathrm{LV})$

-jx(5:KV+1,3:LV)-jy(4:KV,3:LV)+jy $(4: \mathrm{KV}, 2: \mathrm{LV}-1)) ; \quad$ jy $(4: \mathrm{KV}, \mathrm{LV})=0.0$;

end $\mathrm{v}(4: \mathrm{KV}, \mathrm{LV}+1)=\mathrm{jy}(4: \mathrm{KV}, \mathrm{LV}) * \operatorname{mean}(\mathrm{Zl})$;

Zin=((amp 1./amp jy).*exp(-i*arg/2))-((i*arg*vpm/2)*ls1);

$\mathrm{S} 11=(\mathrm{Zin}-\overline{50}) \cdot /(\mathrm{Zin}+50)$;

$\mathrm{S} 11 \mathrm{~m}=\mathrm{abs}(\mathrm{S} 11)$;

$\mathrm{S} 11 \mathrm{~dB}=20 * \log 10(\mathrm{~S} 11 \mathrm{~m})$;

S11a $=$ angle(S11);

$\mathrm{S} 11 \mathrm{ad}=\mathrm{S} 11 \mathrm{a} * 180 / \mathrm{pi}$ 


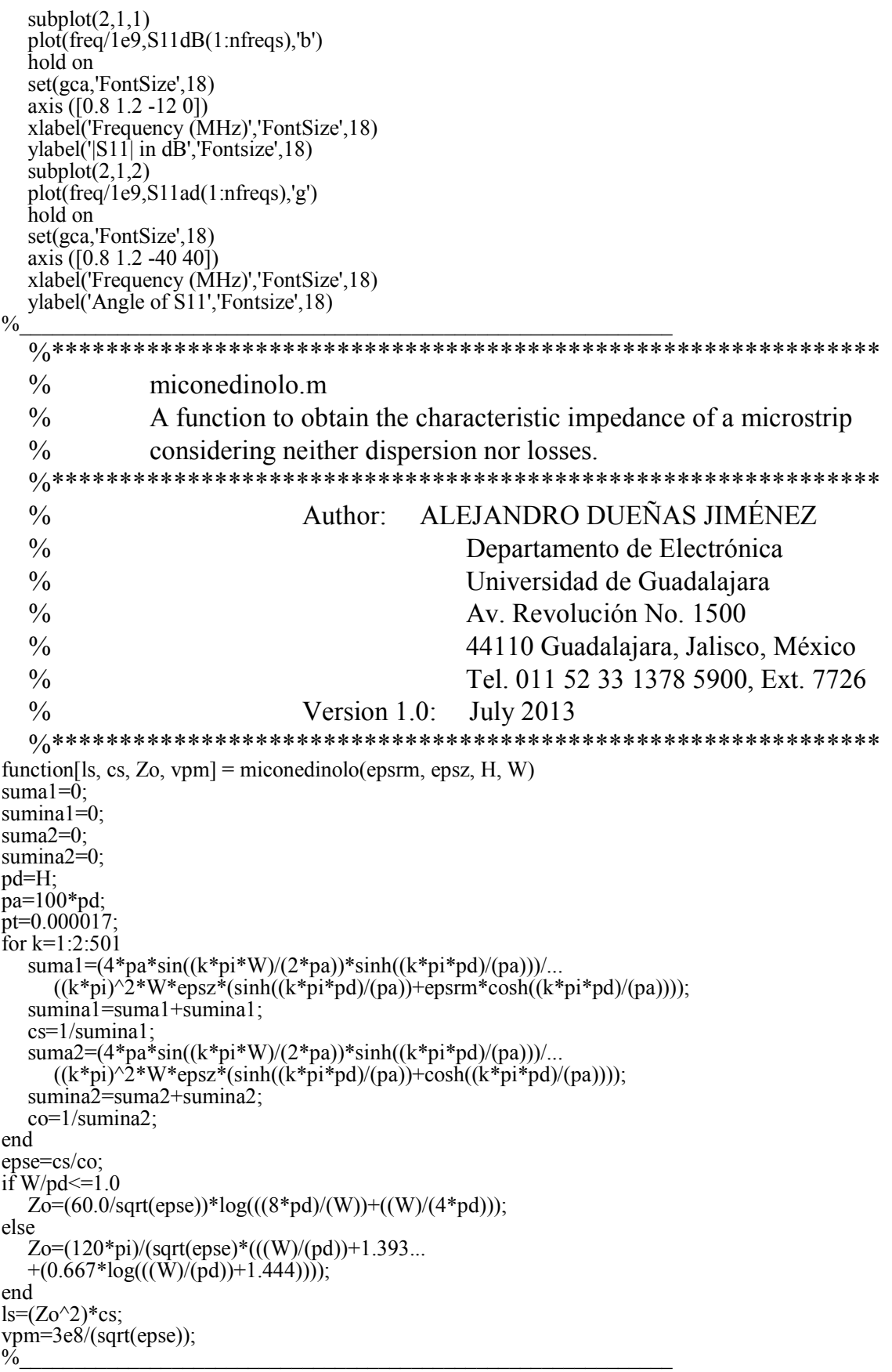

\section{The Simulations}

Table I. Input parameters for the printed wire dipole examples 1 and 2

\begin{tabular}{|c|c|c|}
\hline Printed dipole example & 1 & 2 \\
\hline $\begin{array}{c}\text { Normalized free space } \\
\text { wavelength }\end{array}$ & 4.85 & 2.5 \\
\hline Wire radius in $\lambda_{0}(a)$ & 0.00005 & 0.000025 \\
\hline Resonant length in $\lambda_{0}(L)$ & 0.317 & 0.23 \\
\hline $\begin{array}{c}\text { Substrate dielectric constant } \\
\left(\varepsilon_{r}\right)\end{array}$ & 3.25 & 8.5 \\
\hline $\begin{array}{c}\text { Substrate thickness in } \\
\lambda_{0}(B)\end{array}$ & 0.1016 & 0.15 \\
\hline Time steps & 7000 & 14000 \\
\hline
\end{tabular}

As mentioned at the introduction, the best way to teach electromagnetic simulation is by means of a simple method as the 2D-FDTD. Different to the old empiric analytical techniques [8], [9] and to the very sophisticated methods [10], [11], the 2D-FDTD method offers rapid and confident first approximation simulations that can be useful to feedback other advanced methods [12], [13], [14]. These advantages are profited in the simulations here realized to introduce a real time teaching process. The Table 1 shows the necessary input parameters to simulate two different printed wire dipoles by means of Code1. As in [2], all pertinent dimensions are normalized to the free space wavelength $\lambda_{0}$ (lambda). The input parameters to simulate the third example, 
which consists of a printed planar dipole (Code2) are simply a sweeping of the dipole length, which varies from 3 to 30 in steps of 3 , a normalized substrate thickness of 0.8 , and the time steps to complete the simulation which are 2000 .

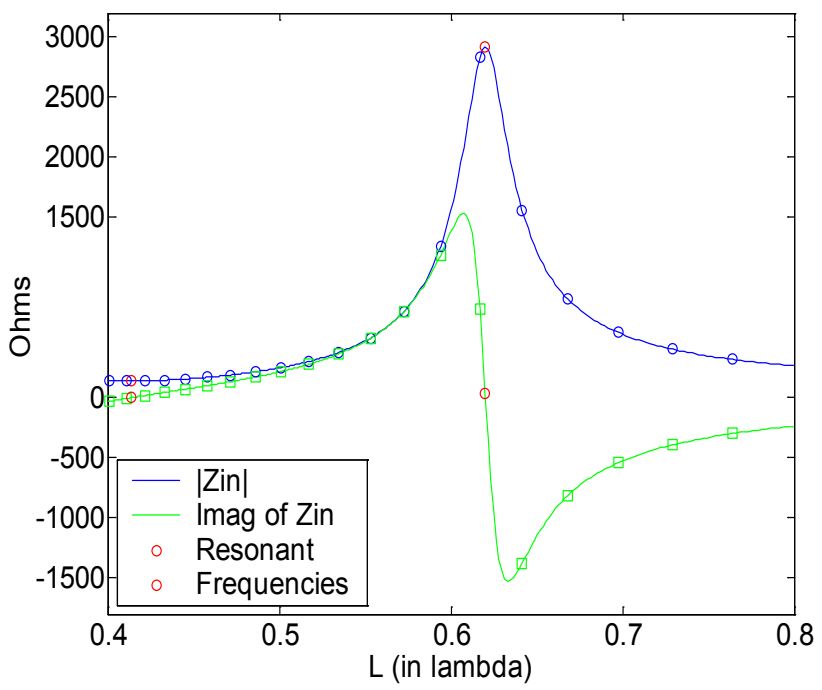

Figure 1. Input impedance versus resonant length (in $\lambda_{0}$ ) for a printed wire dipole with $a=0.00005 \lambda_{0}, L=0.317 \lambda_{0}, \varepsilon_{r}=3.25$ and $B=0.1016 \lambda_{0}$.

The planar dipole has a width of $0.2 \mathrm{~cm}$, a length varying from 12.6 to $14.4 \mathrm{~cm}$, and a substrate dielectric constant of 3 with thickness of $0.05 \mathrm{~cm}$.

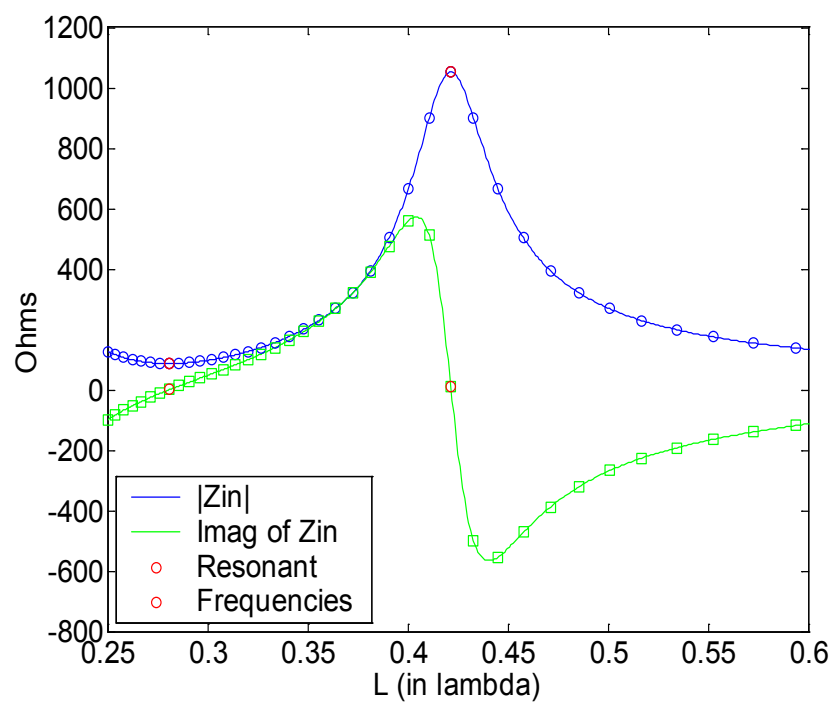

Figure 2. Input impedance versus resonant length (in $\lambda_{0}$ ) for a printed wire dipole with $a=0.000025 \lambda_{0}, L=0.23 \lambda_{0}, \varepsilon_{r}=8.5$ and $B=0.15 \lambda_{0}$.

The responses of the printed wire dipoles are shown in Figs, 1 and 2. As can be seen from Fig. 1 or by running the Code1, the first wire dipole presents a resonant frequency of around $483.75 \mathrm{MHz}$ corresponding to a wavelength of $61.973 \mathrm{~cm}$ on free space. Similarly, from Fig. 2 or by executing once again the Code1, the second wire dipole presents a resonant frequency of around $711.25 \mathrm{MHz}$ corresponding to a wavelength of $42.151 \mathrm{~cm}$ on free space. In these examples the microstrip widths have been considered as the wire radii instead of the diameters, thereby the results are different to those shown in [2]. The responses of the printed planar dipole are shown in Fig. 3.

As can be seen from this figure, the resonant frequency can be found around $950 \mathrm{MHz}$ corresponding to a wavelength of $31.557 \mathrm{~cm}$ on free space.
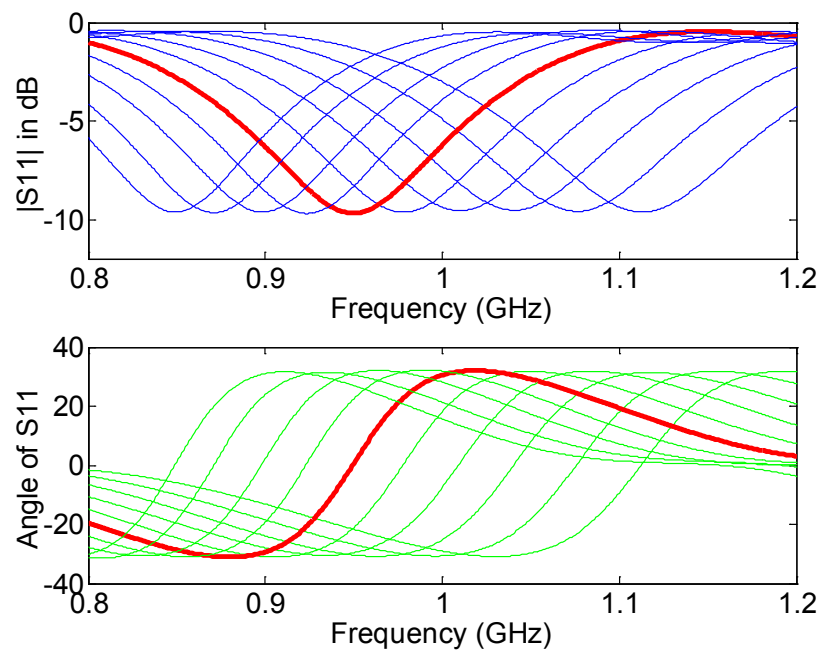

Figure 3. $\mathrm{S}_{11}$ parameter of a printed planar dipole versus frequency. The planar dipole has a $0.2 \mathrm{~cm}$ width, a length varying from 12.6 to $14.4 \mathrm{~cm}$ and a substrate with a dielectric constant of 3 and a thickness of $0.05 \mathrm{~cm}$

\section{Conclusion}

A simple strategy for teaching and learning electromagnetic simulation was presented. The 2D-FDTD method proved to be a good tool for this purpose. Two codes and a function were written in Matlab ${ }^{\circledR}$ language to find the responses of three different microstrip printed dipoles. The algorithms are easy to implement and modify giving very fast responses. However, the user should be careful since an oversimplification of those could generate significant errors, mainly on the more important parameters to be assessed, which are the input impedance and the reflection coefficient

\section{REFERENCES}

[1] A. Dueñas Jiménez, 2-D Electromagnetic Simulation of Passive Microstrip Circuits, CRC Press a Taylor and Francis Company, Boca Raton, Florida, 2009, 274 pp.

[2] I. E. Rana and N. G. Alexopoulos, "Current distribution and input impedance of printed dipoles", IEEE Trans. Antennas and Propagat., vol. AP-29, pp. 99-105, Jan. 1981.

[3] Anon., Using electromagnetic analysis for RFID antenna design, Sonnet Application Note: SAN-206A, Sonnet Software, Inc., 2006.

[4] E. O. Hammerstad, Equations for microstrip circuit design, in 1975 Proceedings of 5th European Microwave Conference, pp. 268-272. 
[5] D. M. Pozar, Microwave Engineering, Reading, Massachusetts: Addison-Wesley, 1990, 726 pp.

[6] A. Dueñas Jiménez, "Frequency- and time-domain simulation of microstrip squares by using 2D-FDTD electromagnetic analyses," published in the International Workshop on Recent Advances in Microwave \& Optical Communication Technology Proceedings, in association with 12th Int. Symp. Microw. Opt. Tech., SRM University, Chennai, India, Dec. 2009.

[7] A. Dueñas Jiménez, "Frequency- and time-domain simulation of microstrip squares by using 2D-FDTD electromagnetic analyses," Int. Journal Microw. Opt. Tech., vol. 5, pp. 1-6, Jan. 2010.

[8] D. A. Huebner, "An electrically small microstrip dipole planar array," in Proc. Workshop on Printed Circuit Antenna Technol., Las Cruces, NM, Oct. 1979, pp. 17.1-17.16.
[9] H. G. Oltman and D. A. Huebner, "Electromagnetically coupled microstrip dipoles," IEEE Trans. Antennas Propagat., vol. AP-29, pp. 152-158, Jan. 1981.

[10] P. P. Silvester and R. L. Ferrari, Finite Elements for Electrical Engineers, Cambridge University Press, Cambridge, 1983.

[11] T. Weiland, A discretization method for the solution of Maxwell's equations for six-component fields, Electronics and Communication (AEU), vol. 31, p. 116, 1977.

[12] R. F. Harrington, Field Computation by Moment Methods, Macmillan, New York, New York, 1968.

[13] A. Taflove and S. C. Hagness, Computational Electrodynamics the Finite-Difference Time-Domain Method, Artech House, Norwood, Massachusetts, 2000.

[14] D. M. Sullivan, "Electromagnetic Simulation Using the FDTD Method, IEEE Press, Piscataway, New Jersey, 2000. 